\title{
News from the EU cluster ICT for Water
}

\author{
Gabriel Anzaldi \\ R\&D Food \& Environment \\ BARCELONA DIGITAL TECHNOLOGY CENTRE - Barcelona (Spain) \\ ganzaldi@bdigital.org
}

Due to growing population and economy, seasonal climatic conditions have changed, including extreme events as floods and droughts. This affects as a whole the availability of water resources at world level. ICT and water efficiency is a key policy issue with potential for new research area that includes decision supporting system for the measurement of water quality and quantity including the recycling and water reuse processes. This necessitates increased interoperability between water information systems at EU and national levels and efficiency of water resources management. Aware of these needs, the European community has promoted various research activities in order to advance in their solution. As a result of these funding actions, an initiative to group projects with common research and innovation interest emerged to enhance their outcomes.

The ICT4WATER (http://ict4water.eu) is a cluster of ICT and water management oriented projects, all co-funded by the European Commission, our common goal is to increase efficiency in water management and enable greater cooperation among water regulators, operators and users by deploying solutions provided by Information and Communication.

The vision of the cluster is to establish and support a thriving and interconnected Information and Communication Technology (ICT) for the Water Community with the main objective of promoting the dissemination and exploitation of the results of European Union (EU) funded activities in this area. Once the project ends, the vision is twofold: in one hand, contribute to advance in the consolidation of an ICT for the Water Community that will be better informed, defined and integrated than today; on the other hand, ICT4WATER will help the results and outcomes from current research projects improving their exploitation plans and increasing their dissemination potential by delivering co-produced knowledge to a wide range of stakeholders and actors within the water community.

The target community will be mainly composed of different stakeholders including Water Authorities, Water Operators, System Integrators, ICT for Water technologies professionals, Policy Makers, and the relevant industry at large. The academic and research community will be also targeted and strongly represented in the cluster. Considering the above and to concretise, the following groups shall benefit from the cluster initiatives:

- $\quad$ Participants of the EU funded activities: i) CIP-ICT-PSP thematic network @qua focused on ICT for water efficiency; ii) FP7-ICT-2013.6.3 projects: WatERP, iWIDGET, EFFINET, UrbanWater, ICeWATER, WISDOM,
DAIAD, ISS-EWATUS, SmartH2O, Waternomics and iii) other relevant ICT for Water EU funded projects, such as the new H2020 Water-4a winners: WIDEST, FREEWAT, KINDRA, WaterInnEU and BlueSCities (which are going to became new members of the cluster). From which outputs will contribute feeding main project contents, knowhow, and objectives.

- Members of significant international associations like: i) IWA with 10,000 individual and 500 corporate members in 130 different countries, including scientists from across many disciplines, economists, accountants, social scientists, and managers and leaders among those professions; and ii) WSSTP with 98 members and a network of more than 700 individuals from industry, research, technology providers, policy makers and water users.

- The stakeholder network of ICT for the Water Community, including academic and research communities, industry, water technologies professionals, Small and Medium Enterprises (SMEs), start-ups and the public at large, end users and customer organisations.

- Decision makers trying to identify the most promising outcomes from research and innovation research areas.

These are the main objectives of the current projects integrating the cluster and those which are going to became members:

- $\quad$ EFFINET (Founder member) - http://effinet.eu/ - to improve the efficiency of drinking water networks by managing better consumer behaviour via advanced metering, monitoring of user demand profiles, fault detection and predictive control techniques and integration of information stemming from various sources. Validation: Barcelona (ES) and Lemesos (CY).

- ICeWater (Founder member) - http://www.icewater-project.eu - to develop infrastructure for smart metering and real-time monitoring of water infrastructures, with the aim of lowering consumption during peak periods, detecting and localising leakages in real-time and optimising the water-energy nexus. Validation : Milan (IT) and Timisoara (RO).

- iWIDGET (Founder member) - http://www.i-widget. eu - to develop specialized software ("widgets" for the iWIDGET system), in order to make households more aware of their water and water-related energy consumption patterns. The project also aims to help utilities and ICT industry with the sharing of information in order to improve their demand forecasting capacities and opera- 
tional efficiency, while also contributing to a sustainable partnership of ICT and water domain stakeholders. Validation: Barcelos (PT), Southern Water region (UK) and a pilot of volunteers in Athens (GR)

- UrbanWater (Founder member) - http://urbanwater-ict. eu - to integrate advanced metering solutions, real-time communication of consumption patterns with predictive capability, adaptive pricing and customer empowerment tools. Validation: urban setting in Scotland (UK) and Algarve (PT).

- WatERP (Founder member) - http://www.waterp-fp7.eu/ - to develop a web-based, open management platform to enable water supply distribution chains to be managed in a coordinated and customised way, based on open interfaces and standards. The aim is to improve coordination among water management actors and to foster behavioural change in order to reduce water and energy consumption. Validation: Llobregat river basin (ES) and Leipzig water supply network (DE).

- DAIAD - http://daiad.eu -will focus on real-time knowledge of residential water consumption. The goal is to research and develop innovative low cost, inclusive technologies for real-time, high granularity water monitoring and knowledge extraction to incur behavioural changes, water demand strategies and water pricing. Validation : Athens (GR) and Brighton (UK)

- ISS-EWATUS - http://issewatus.eu - will increase the awareness of water consumption via social media platform (smartphones, tablets) to reduce water consumption and will build a decision support system to reduce leaks. Coupling with adaptive pricing policy and reduction of peaks. Validation : Skiatos (GR); Sosowiec (PL)

- SmartH2O - http://www.smarth2o-fp7.eu - aims at providing an ICT enabled platform to design, develop and implement better water demand management in collecting user behavioural data due to smart meters and an online social participation application (social game). Awareness campaigns and price signals are delivered through the same app to inform the users on how to save water and money. Validation : Gordola (CH) and London (UK).

- WATERNOMICS - http://waternomics.eu - will enable the introduction of Demand Response principles and open business models through an innovative human centric approach that uses personalized water data, water availability based pricing, and gamification of water usage statistics. Validation: Thessaloniki (GR), Milano (IT) and Sochaczew (PL)

- WISDOM - http://www.wisdom-project.eu/ - aims to; (a) improve demand management by increasing user awareness, achieving a significant reduction of total and peak water consumption, (b) improve resource management by achieving a reduction in water network energy usage and improved resource efficiency and (c) improve the business operations of water utilities by enabling the collection of more detailed network knowledge using semantic and crowd-sourcing techniques. WISDOM will be validated in pilots in Cardiff (UK) and La Spezia (IT).

- BlueSCities (Expected member) The project Blueprints for Smart Cities aims to develop the methodology for a coordinated approach to the integration of the water and waste sectors within the 'Smart Cities and Communities' EIP. It will identify synergies in accordance with Smart City ideology and compliment other priority areas such as energy, transport and ICT. It will seek to contribute to the achievement of the 20-20-20 objectives.

- $\quad$ FREEWAT (Expected member) aims at promoting water management and planning by simplifying the application of the Water Framework Directive and other EU water related Directives. FREEWAT will be an open source and public domain GIS integrated modelling environment for the simulation of water quantity and quality in surface water and groundwater with an integrated water management and planning module.

- KINDRA (Expected member) The overall objective of KINDRA is to create an inventory of this knowledgebase and then use the inventory to identify critical research challenges in line with the implementation of the WFD and new innovation areas within integrated water resources management based on the latest research.

- WaterInnEU (Expected member) primary vision is to create a marketplace to enhance the exploitation of EU funded ICT models, tools, protocols and policy briefs related to water and to establish suitable conditions for new market opportunities based on these offerings. WaterInnEU will build upon existing knowledge bases and platforms developed in previous projects but will provide new concepts, connections, and components that are essential for a marketplace to work.

- WIDEST (Expected member) the project has the vision of establishing and supporting a thriving, interconnected ICT for water community to promote the dissemination and exploitation of EU funded activities and results in this area. WIDEST will address its goals through a project-to-project approach and the coordination among relevant stakeholders by means of five objectives.

In particular WIDEST coordination and support action, assumes the challenge of promoting this community. Indeed, there is a need to promote the dissemination and exploitation of EU funded activities, including relevant ICT for Water Technologies. The various mechanisms within the WIDEST project will help researchers and the rest of stakeholders by making them aware of each other, encouraging collaboration and ensuring wider application of innovative solutions. By identifying fruitful (and fruitless) directions, as well as by establishing sustainable foundations through an EU-EIP for Water Smart Water Action Group, WIDEST aims to foster more efficient and impactful communication within the du- 
ration of the program and well beyond it, by means of a selfsustainable community building in the area of ICT for Water Systems and Applications

This vision entails the following particular ten goals:

1. To aggregate and promote ICT for Water management projects by developing an updated portfolio of promising and disruptive innovations for effective technologies of varying readiness levels, contributing to the implementation of the "European Innovation Partnership on Water - Strategic Implementation Plan";

2. Support discussion and collaboration among key academic and commercial stakeholders;

3. Encourage a sustainable collaboration infrastructure, including ICT communities, water stakeholders and policy makers;
4. Foster ICT for Water and ICT standardization efforts regarding to semantic interoperability and ontologies;

5. Contribute to identify gaps/barriers/bottlenecks on existing regulations blocking innovations and smart technologies;

6. Contribute to the achievement of EIP "Smart technologies" and "Decision Support System and Monitoring" priorities objectives;

7. Advise on needs for policy development for increasing efficiency of funding smart technologies;

8. Develop roadmap for effective implementation of a holistic approach of ICT tools through 2020 [2];

9. Disseminate knowledge and strategic objectives to the water community at large;

10. To enhance implementation, interoperability and standardization, together with bringing economy of scale and business opportunities to the already existing solutions on the projects portfolios.

\section{ictlwater.eu}

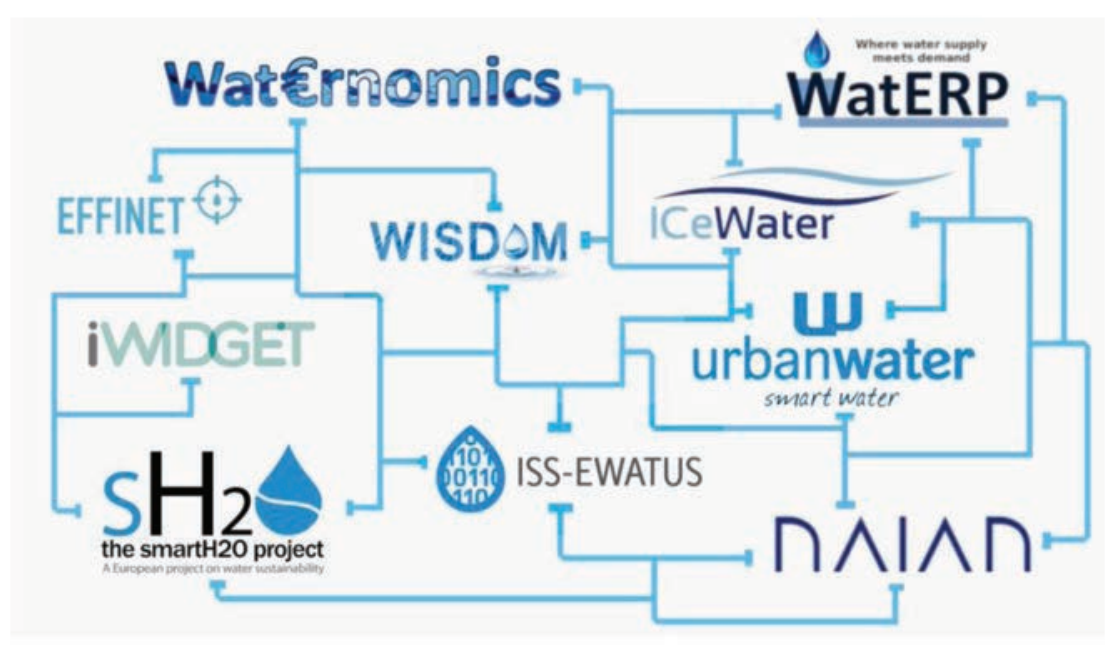

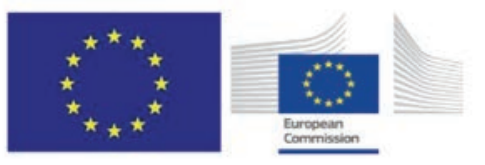

These projects have received funding from European Union's Seventh and H2020 Framework Programmes from research, technological development and demonstration. 